\title{
A MWPC AS AN ENERGY LOSS DETECTOR FOR THE ISR
}

\author{
H. FREHSE, M. HEIDEN*, M. PANTER and F. PIUZ
}

CERN, Geneva, Switzerland

\begin{abstract}
A MWPC providing position and energy loss measurement is described. One aim of the detector is to identify background electrons in a multitrack event at the CERN ISR (Intersecting Storage Rings). The chamber consists of four gaps of $2 \times 0.5 \mathrm{~cm}$ each giving a combined energy resolution for minimum ionizing particles of $50 \%$ fwhm. The 500 wires are equipped with fast charge amplifiers allowing the measurement of the charge up to a rate of $6 \mathrm{kHz}$ per wire. The dynamic range covered linearly is $0.05-16 \mathrm{pC}$. Corrections due to the inclination of the tracks (saturation effect) are applied.
\end{abstract}

\section{Introduction}

A multiwire proportional chamber (MWPC) measuring position and energy loss of charged particles has been developed for the Split Field Magnet (SFM) spectrometer, installed at the CERN ISR.

The chamber is a part of an electron telescope designed to trigger on single electrons. Background electrons originate mainly from $\pi^{0}$ Dalitz decay and pair production by photons in the ISR vacuum chamber. Close $\mathrm{e}^{+} \mathrm{e}^{-}$with unresolved trajectories can be rejected by their high apparent energy loss, whereas asymmetric pairs are recognized by positive identification of the low momentum partner of the pair.

\section{Constraints}

The design of the chamber has to satisfy the following constraints:

a) Energy resolution must allow separation of particles losing, on the average, 1.5-4 times the mean energy loss of minimum ionizing particles: $\times 2$ in the case of unresolved pair with respect to single electron; $\times 1.5$ to 4 when the analysis of low momenta pions is needed.

b) To cover the required solid angle $(2 \mathrm{sr})$, the chamber is situated close to the intersection $(15 \mathrm{~cm})$; this implies:

- a construction technique demanding for a minimum of material,

- no electronics fixed to the chamber for accessibility,

- a large variation of the angle of incidence of the trajectories: $0-60^{\circ}$,

- two to three particles accompanying, on the av-

* Visitor from Heidelberg University. erage, the electron (or unresolved pair) with sufficient localisation in the chamber,

- a particle rate of $2-6 \mathrm{kHz}$ per wire.

\section{Chamber construction - spatial and energy resolution}

The chamber is built as a stack of foam sandwich plate combining low density together with thin frames ${ }^{1,2}$ ). The main features of the chamber are listed below:

- sense wire plane 4 ,

- plane sequence seen

from the intersection (Vert./V/Horiz./Vert.),

- half gap

- wire spacing (opti-

cally controlled) $\quad(4 \pm 0.05) \mathrm{mm}$,

- wire diameter $20 \mu \mathrm{m}$ (Sylvania),

- total gas thickness

(perpendicular to the

plates) $40 \mathrm{~mm}$,

- total chamber thick-

ness $\quad 90 \mathrm{~mm}$,

- total density $\quad 0.8 \mathrm{~g} / \mathrm{cm}^{2}$,

- frame thickness $\quad 5 \mathrm{~mm}$ (vetronite),

- sensitive area $\quad 572 \times 272 \mathrm{~mm}^{2}$,

- number of channels 493,

- gas composition argon/isobutan/methylal

$55 / 38 / 7 \%$ in volume.

The expected spatial resolution is $0.9 \mathrm{~mm}^{3}$ ).

The combined energy resolution of four gaps, measured at the ISR with pions having a momentum spread of $200 \mathrm{MeV} / \mathrm{c}$ to $1000 \mathrm{MeV} / \mathrm{c}$, is $50 \%$ fwhm (sect. 7.1).

The homogeneity of the amplification including electronics was checked, by measuring the peak position of the $5.9 \mathrm{keV}$ line of a ${ }^{55} \mathrm{Fe}$ source. A variation of $\pm 5 \%$ was found. 


\section{Range of the energy loss to be recorded}

Let us call $E_{0}$ the mean energy lost by a minimum ionizing particle traversing $10 \mathrm{~mm}$ of gas mixture. One has to be prepared to record pulses corresponding to the quantity $E_{0}$, multiplied by the following factors:

$-\times 4$ for energy separation purpose,

$-\times 3$ to record events belonging to the higher part of the spectrum, $>E_{0}$,

$-\times 2$ for the increased path length of inclined tracks traversing one wire cell (see the figure in sect. 7.2).

That is, in the extreme case, $E_{\max } \approx 25 E_{0}$. On the other hand, $E_{0}$ will be divided by:

- :3 to record events belonging to the lower part of the spectrum, $<E_{0}$,

$-: 3$ for inclined tracks traversing partially several wire cells.

That is, in the worst case, $E_{\min } \approx 0.1 E_{0}$. One estimates then the energy range to be approximately $250 E_{\min }$.

\section{Electronics}

The design criteria of the electronics are:

- Energy range to be recorded which prescribes the needed linear range of the amplifier together with a minimum equivalent noise level.

- The SFM event trigger and the particle rate per wire which influence the shape of the analog signal.

- The possible generation of a Fast OR signal (FOR) per group of wires for geometry trigger purpose.

\subsection{SFM EVENT TRIGGER ${ }^{4}$ ) AND PARTICLE RATE}

The analog signals are recorded in charge sen- sing ADCs*. Their reading gate is generated by the SFM event trigger which determines the timing. The time needed for the trigger decision is $500 \mathrm{~ns}$ which is the maximum time available for the generation of a stable analog level. In addition, the typical time jitter of proportional chambers of $100 \mathrm{~ns}^{+}$requires that this level has to stay constant for this time. On the other hand, the rate per wire (maximum $6 \mathrm{kHz}$ ) imposes a maximum duration time of the analog pulse in order to avoid overlap of pulses.

\subsection{Electronics Chain (fig. 1)}

A charge preamplifier ${ }^{5}$ ) is connected to a wire of the chamber by $4.5 \mathrm{~m}$ of thin coaxial cable ${ }^{+}$ $(\varnothing=1.3 \mathrm{~mm})$. It has a balanced output which feeds a receiving circuit through $65 \mathrm{~m}$ of twisted pair cable, situated in the counting room. At this place, gain and dc level can be adjusted. This circuit provides: (a) an analog output sent after attenuation $(\times 6)$ (sect. 6.1) to the ADC channel, allowing the use of short reading gate ( $25 \mathrm{~ns})$, and (b) a FOR output per 12 channels which requires for a clean generation a non-attenuated analog pulse. The main features of these elements are indicated in table 1.

The output linearity of the chain (5\% deviation) extends over a range of $+0.7 \mathrm{~V}$ to $-5 \mathrm{~V}$, equivalent to $16 \mathrm{pC}$. This linear behaviour is kept for any integration time included between 20 and 200 ns. However, at a fixed input charge, an output loss of $5 \%$ is observed when the integration

* LRS 2249A 12 channels.

+ This number is a maximum, as the longest collection time of primary ionisation is $100 \mathrm{~ns}$ (half gap: $5 \mathrm{~mm}$ ).

$\uparrow$ FILOTEC VMTX50.

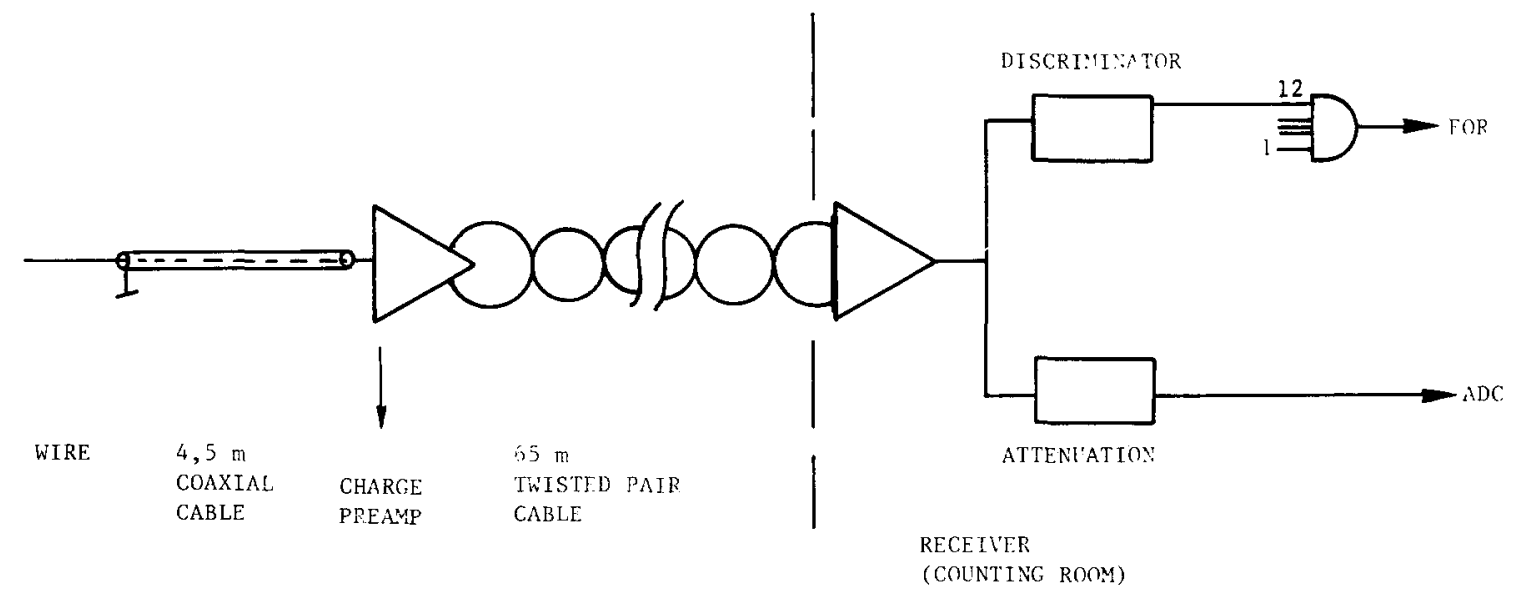

Fig. 1. Sketch of the electronic chain. 
TABLE 1

Main features of the electronics chain.

\begin{tabular}{lcc} 
& Preamplifier & $\begin{array}{c}\text { Receiver } \\
\text { output }\end{array}$ \\
\hline Sensitivity $(\mathrm{mV} / \mathrm{pC})$ & 100 & 300 \\
Equivalent noise $(\mathrm{pC})$ & $5 \times 10^{-3}$ & $(3-4) \times 10^{-2}$ a \\
Decay time $(\mu \mathrm{s})$ & & 1.5 \\
Equivalent FOR threshold $(\mathrm{pC})$ & & $0.1-0.2^{\text {a }}$ \\
\hline
\end{tabular}

a Measured at the ISR.

time reaches $400 \mathrm{~ns}$. As the chamber half gap $(5 \mathrm{~mm})$ corresponds to a collection time of the primary ionisation of $100 \mathrm{~ns}$, the preamplifier operates in a fully linear way.

As seen in fig. 2, the pulse shape delivered at the receiver output shows a sufficiently long "flat top" (small amplitude variation over $100 \mathrm{~ns}$ ) occurring after a suitable time for the SFM trigger, smaller than $500 \mathrm{~ns}$.

Exposing the chamber to a high particle flux (the ISR running condition: $\sim 6 \mathrm{kHz}$ per wire), it has been checked that the pile-up of pulses does not spoil the performance quoted in sect. 3 .
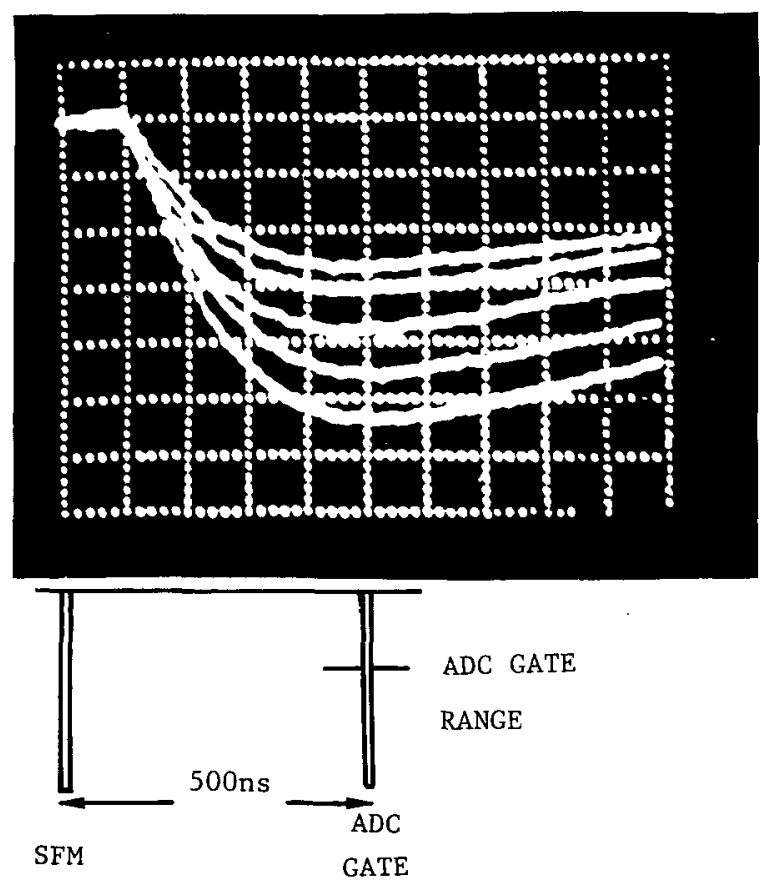

TRIGGER

Fig. 2. Pulse shape at the receiver output. $100 \mathrm{~ns}$ per division. The pulse is situated, in time, with respect to the SFM trigger and the $\mathrm{ADC}$ gate.
Finally, let us remark that for this special application, our goal is fulfilled with a simple charge preamplifier of moderate cost: 20 SF per two channels produced in a semi-hybridation technique* (300 pieces ordered). The remaining cost of the chain (50 SF) consists in the receiver and fast OR circuitry.

\subsection{Electronics calibration}

A test pulse, capacitatively coupled to the inputs of a preamplifier module ( 30 channels), is used to adjust the electronic gain of each channel. These calibration parameters are recorded and later on, used in the off-line analysis.

\section{Working conditions at the ISR}

\subsection{Chamber}

At the chosen operating voltage of $2.3 \mathrm{kV}$, a mean charge of $0.4 \mathrm{pC}$ has been measured ${ }^{6}$ ) for an energy loss $E_{0}$ (sect. 4). This measurement was performed using the same gas mixture, with particles of fixed momentum and trajectory. Under these conditions, the gas amplification is $(2 \pm 0.2) \times 10^{4}$. With the electronic parameters quoted in the sect. $5, E_{0}, E_{\min }$ and $E_{\max }$ will respectively correspond to the ADC channels 40,4 ,

* Manufacturer: C.I.T. AlCATEL.

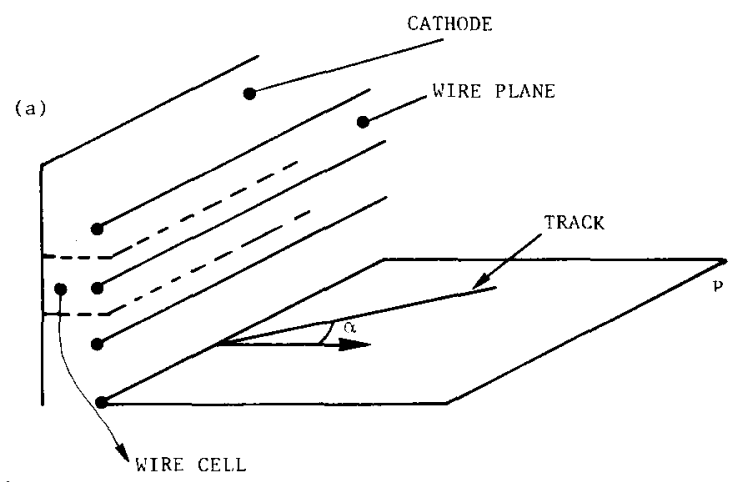

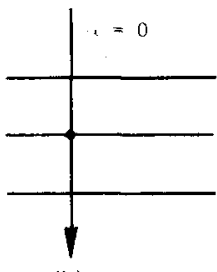

(b)

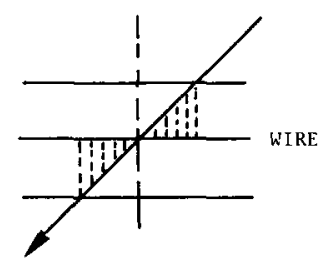

(c)
Fig. 3. (a) Definition of the angle $\alpha$. The track lies in the plane $P$, which is orthogonal to the sense wire plane. (b) $\alpha=0$, maximum of saturation: all the primaries are collected at nearly the same place on the wire. (c) $\alpha$ large, no saturation. The primaries are collected along a large distance on the wire. 
1000 (above pedestal) or charges $0.4,0.04,10 \mathrm{pC}$. These values lie inside the linear range of the electronics or the ADC range.

\subsection{Monitoring}

A low rate $\gamma$ source $\left({ }^{55} \mathrm{Fe}\right)$ fixed on the chamber, together with atmospheric pressure recording, allow a check of the long term stability of the system.

\section{Off-line analysis}

\subsection{COMBINED ENERGY RESOlution}

A sample of electron candidates recorded with the SFM by the ISR experiment R416 ${ }^{7}$ ) was used for the analysis. The tracks of triggering electrons were reconstructed ${ }^{8}$ ) and the corresponding wire clusters in the $\mathrm{d} E / \mathrm{d} x$ chamber determined. The pulse heights of the wires hit by the reconstructed tracks were corrected (sect. 7.2) and then summed up in each plane. After these corrections, the energy loss measurements of the four gaps were available for the reconstructed tracks. The measurement showing the highest energy loss was excluded and the mean of the remaining three measurements was taken. This method was found to give the best resolution for the combined energy loss measurements.

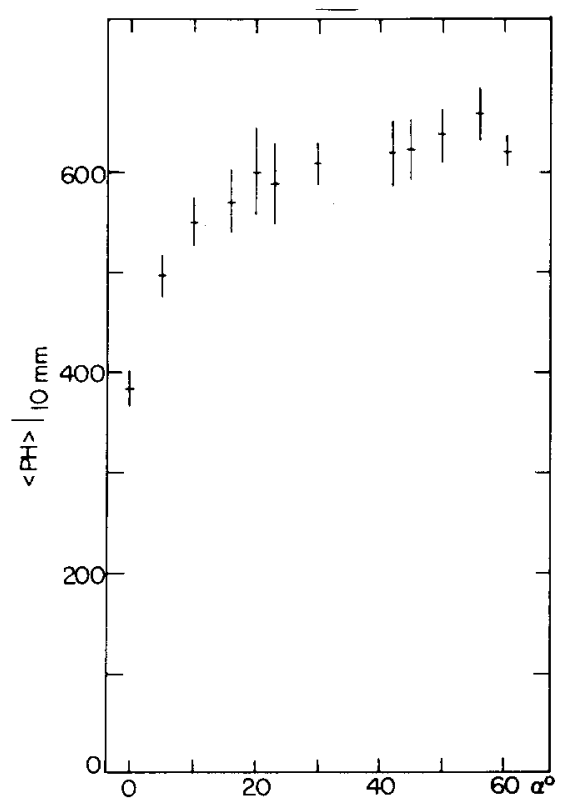

Fig. 4. Mean of pulse height spectrum normalised to $10 \mathrm{~mm}$ path length plotted vs the angle $\alpha$. Experimental conditions: angle definition: $\pm 0.3^{\circ}$; tracks lie in a plane $P$; measurements on one wire with $\pi^{-} 1.4 \mathrm{GeV} / c$; half gap: $5 \mathrm{~mm}$; pitch $4 \mathrm{~mm}$; hv: $2.3 \mathrm{kV}$; gas amplification: $2 \times 10^{4}$.

\subsection{CORRECTION ON PULSE HEIGHTS}

The first corrrection, related to the electronic gain variation, uses the information described in sect. 5.3. The second correction is connected to the inclination of the track.

Let us consider the situation (fig. 3) of a track traversing one wire cell and making an angle $\alpha$ with the normal to the wire. When this angle equals to zero, the avalanche process occurs at a very local spot on the wire $(\sim 100 \mu \mathrm{m})$. Depending on the gas multiplication, a saturation in the pulse height is observed ${ }^{9}$ ) compared to the situation of large $\alpha$ where the avalanches are distributed along the wire (millimeters) and the pulse height does not depend then on the angle $\alpha$.

This behaviour has been studied ${ }^{6}$ ) under well defined conditions of momentum and angles for different gas amplification and gas thickness. From these results, fig. 4 shows the pulse height dependence on $\alpha$ for the same working conditions as used at the ISR. This curve indicates the mean correction factor to be applied to an energy loss measurement in a gap where the angle $\alpha$ has been preliminarily calculated. By taking the pulse height measured at $\alpha=0^{\circ}$ as unity, the maximum correction reaches 1.6 for $\alpha \gtrsim 30^{\circ}$ for a gas amplification of $2 \times 10^{4}$.

\subsection{RESULT ON UNRESOLVED TRAJECTORIES}

Fig. 5. shows the distribution of the combined energy loss measurements described in sect. 7.1. One sees clearly two distinct peaks, which corre-

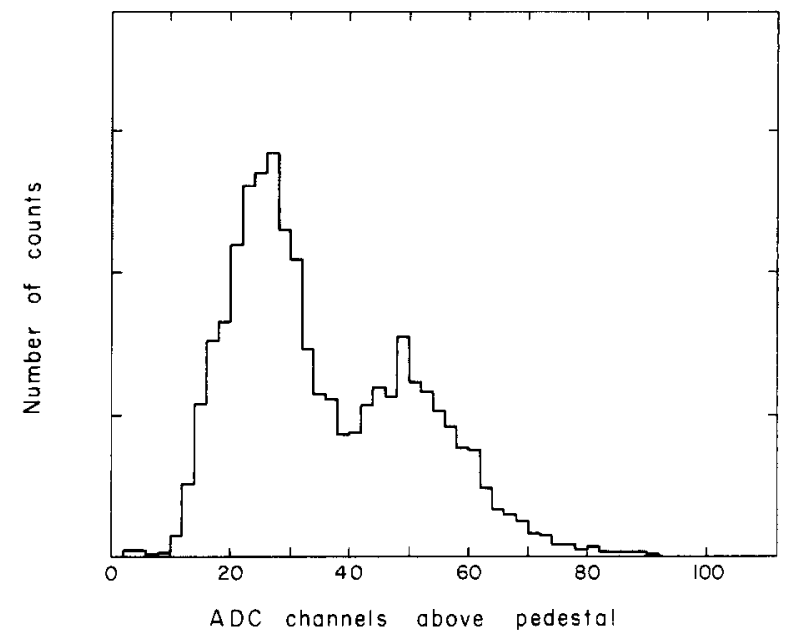

Fig. 5. Pulse height distributions in the $d E / d x$ chamber at the ISR obtained with the electron trigger. The energy loss measurements are normalised to $\alpha=0^{\circ}$. The first peak corresponds to one electron; the second to unresolved electron pairs. 
spond to electrons and not resolved close electron pairs. The first peak is slightly smeared by a background contamination of pions $(\approx 20 \%)$ which have a lower pulse height than the relativistic electrons. Furthermore, the two peaks are widened by the rather big momentum spread of the electrons $(200-1000 \mathrm{MeV} / \mathrm{c})$ which has not been corrected for.

\section{Conclusions}

We have shown that energy loss measurement with MWPC is possible under the stringent constraints imposed by the vicinity of an ISR intersection. Even with a rather small system, a resolution adequate for our application can be achieved.

In comparison to a photomultiplier scintillator technique, a MWPC has the following advantages:

- no sensitivity to strong magnetic fields,

- comparably less material,

- possibility to disentangle the energy loss of different particles emitted into a rather small solid angle because of the good spatial resolution of MWPC.

We are indebted to H. G. Fischer who has initiated this work. We wish to thank E. E. Kluge for his participation.

We acknowledge J. C. Santiard for his major contribution to the electronic design and the continuous technical help from R. Alberganti, J. Cavalli, E. Chesi, M. Dalbignat, G. Di Tore, L. Dumps, P. Strobach and J. Vinzia.

\section{References}

1) R. Bouclier, G. Charpak, E. Chesi, L. Dumps, H. G. Fischer, H. J. Hilke, P. G. Innocenti, G. Maurin, A. Minten, L. Naumann, F. Piuz, J. C. Santiard and O. Ullaland, Nucl. Instr. and Meth. 115 (1974) 235

2) R. Bouclier, R. C. A. Brown, E. Chesi, L. Dumps, H. G. Fischer, P. G. Innocenti, G. Maurin, A. Minten, L. Naumann, F. Piuz and $O$. Ullaland, Nucl. Instr. and Meth. 125 (1975) 235

3) H. G. Fischer, F. Piuz, W. J. Schwille, G. Sinapius and O. Ullaland, Proc. Int. Meeting on Proportional and drifi chambers, Dubna (1975).

4) W. Bell, E. Chesi, R. Cooper, H. G. Fischer, G. Flügge, H Frehse, Ch. Gottfried, B. Heck, P. G. Innocenti, I. McLaren, A. Minten, M. Scirè and D. Wegener, Nucl. Instr. and Meth. 124 (1975) 437.

5) J. C. Santiard, Charge amplifier in semi-hybridation, EP Int. Note (7 October 1976); B. Righini and J. Schiari, Test of charge amplifier for MWPC, EP Report no. 53. (January 1978).

6) F. Lapique and F. Piuz, these proceedings.

7) Experiment R416: Study of rare events at the Split Field Magnet, CERN-Collège de France-Heidelberg-Karlsruhe Collaboration (CCHK).

8) D. Drijard and A. Norton, New SFM software, Documentation in preparation.

9) A. Breskin, G. Charpak, F. Sauli, M. Atkinson and G. Schultz, Nucl. Instr. and Meth. 124 (1975) 189. 